Pacific Journal of Mathematics

LOCAL BEHAVIOUR OF AREA FUNCTIONS OF CONVEX 


\title{
LOCAL BEHAVIOUR OF AREA FUNCTIONS OF CONVEX BODIES
}

\author{
WILLIAM J. FIREY
}

The area function of a convex body $K$ in Euclidean $n$-space is a particular measure over the field $\mathscr{B}$ of Borel sets of the unit spherical surface. The value of such a function at a Borel set $\omega$ is the area of that part of the boundary of $K$ touched by support planes whose outer normal directions fall in $\omega$. In particular the area function of the vector sum $K+t E$, where $t$ is nonnegative and $E$ is the unit ball, is a polynomial of degree $n-1$ in $t$ whose coefficients are also measures over $\mathscr{B}$. To within a binomial coefficient, the coefficient of $t^{n-p-1}$ in this polynomial is called the area function of order $p$. For $p=1$ and $p=n-1$ necessary and sufficient conditions for a measure over $\mathscr{B}$ to be an area function of order $p$ are known, but for intermediate values of $p$ only certain necessary conditions are known. Here a new necessary condition is established. It is a bound on those functional values of an area function of order $p$ which correspond to special sets of $\mathscr{B}$. These special sets are closed, small circles of geodesic radius $\alpha$ less than $\pi / 2$; the bound depends on $\alpha, p$ and the diameter of $K$. This necessary condition amplifies an old observation: area functions of order less than $n-1$ vanish at Borel sets consisting of single points.

To examine area functions in detail, we write $\Pi(u)$ for the support plane to $K$ whose outer normal direction corresponds to the point $u$ on the unit spherical surface $\Omega$. For $\omega$ in $\mathscr{B}$ set

$$
B(\omega)=\bigcup_{u \in \omega}(\Pi(u) \cap K) .
$$

The area function of $K$ at $\omega$ is the $(n-1)$-dimensional measure of $B(\omega)$; we denote this by $S(K, \omega)$. $S(K+t E, \omega)$ is a polynomial of degree $n-1$ in $t$; the coefficient of

$$
\left(\begin{array}{c}
n-1 \\
p
\end{array}\right) t^{n-p-1}, \text { where }\left(\begin{array}{c}
n-1 \\
p
\end{array}\right)=\frac{(n-1) !}{p !(n-p-1) !}
$$

is the area function of order $p$ at $\omega$ and is written $S_{p}(K, \omega)$. In particular

$$
S_{n-1}(K, \omega)=S(K, \omega), S_{0}(K, \omega)=S(E, \omega) .
$$

If at each boundary point of $K$ there is a unique outer normal 
$u$ and principal radii of curvature $R_{1}(u), \cdots, R_{n-1}(u)$ and if $\left\{R_{1}, \cdots, R_{p}\right\}$ signifies the $p^{\text {th }}$ elementary symmetric function of these radii, then

$$
S_{p}(K, \omega)=\int_{\omega}\left\{R_{1}, \cdots, R_{p}\right\} d \omega /\left(\begin{array}{c}
n-1 \\
p
\end{array}\right) .
$$

For general convex bodies the total area of order $p$ is a special mixed volume; in detail

$$
S_{p}(K, \Omega)=n V(\underbrace{K, \cdots, K}_{p}, \underbrace{E, \cdots, E}_{n-p}) .
$$

Let $v$ be any fixed point on $\Omega$ and let $\omega_{\alpha}$ be the set of $u$ on $\Omega$ for which

$$
(u, v) \geqq \cos \alpha, 0<\alpha<\pi / 2,
$$

where $(u, v)$ denotes the inner product of $u$ and $v$. We shall prove that

$$
S_{p}\left(K, \omega_{\alpha}\right) \leqq A D^{p} \sin ^{n-p-1} \alpha \sec \alpha=A D^{p} f_{p}(\alpha),
$$

for $p=1,2, \cdots, n-1$, where $D$ is the diameter of $K$ and $A$ depends neither on $\alpha$ nor on $K$.

A. D. Aleksandrov [1] and W. Fenchel and B. Jessen [3] introduced such area functions. They showed that for a measure $\Phi$ over $\mathscr{B}$ to be an area function of order $n-1$, it is necessary and sufficient that, for any $u^{\prime}$

$$
\int_{\Omega}\left(u^{\prime}, u\right) \Phi(d \omega(u))=0, \int_{\Omega}\left|\left(u^{\prime}, u\right)\right| \Phi(d \omega(u))>0,
$$

where these are Radon integrals. Aleksandrov showed also that (2), while necessary for $\Phi$ to be a $p^{\text {th }}$ order area function when $p<n-1$, are not sufficient. In part this depended on the observation that

$$
S_{p}(K,\{v\})=0
$$

for each $v$ on $\Omega$ and $p<n-1$. By letting $\alpha$ tend to zero, we see that (3) is a consequence of (1).

Necessary and sufficient conditions for $\Phi$ to be an area function of order one are given in [4] and [5]. Inequality (1) for $p=1$ was proved in the latter paper and plays a significant part. Items of background are in these papers and [2] and [3].

1. We first show that if (1) holds for convex polyhedra, then it is true for all convex bodies.

Given any convex body $K$ we can find convex polyhedra $K_{m}, m=$ 
$1,2, \cdots$, which approximate $K$ to within $1 / m$ in the sense of the metric

$$
\delta\left(K, K_{m}\right)=\max _{u \in \Omega}\left|H(u)-H_{m}(u)\right|,
$$

where $H$ and $H_{m}$ are the support functions of $K$ and $K_{m}$. For the diameters $D$ and $D_{m}$ of these bodies we have

$$
\lim _{m \rightarrow \infty} D_{m}=D \text {. }
$$

Let $\varepsilon>0$ be such that $\alpha+\varepsilon<\pi / 2$; denote by $\eta_{\varepsilon}$ the open set of $u$ on $\Omega$ for which

$$
(u, v)>\cos (\alpha+\varepsilon)
$$

Clearly

$$
\omega_{\alpha} \subset \eta_{\varepsilon} \subset \omega_{\alpha+\varepsilon} \cdot
$$

By Theorem IX of [3], $S_{p}\left(K_{m}, \omega\right)$ converges weakly to $S_{p}(K, \omega)$ as $m$ tends to infinity. This implies [3, p. 8] that

$$
\liminf _{m \rightarrow \infty} S_{p}\left(K_{m}, \eta_{\varepsilon}\right) \geqq S_{p}\left(K, \eta_{\varepsilon}\right) \geqq S_{p}\left(K, \omega_{\alpha}\right)
$$

since $\eta_{\varepsilon}$ is open. We have used (4) and the monotonicity of $S_{p}(K, \omega)$ in $\omega$ for the final inequality.

Also from (4), the monotonicity of $S_{p}$, and the assumption of (1) for polyhedra, we get

$$
S_{p}\left(K_{m}, \eta_{\varepsilon}\right) \leqq A D_{m}^{p} f_{p}(\alpha+\varepsilon) .
$$

Hence, because $D_{m}$ tends to $D$, (5) and (6) yield

$$
S_{p}\left(K, \omega_{\alpha}\right) \leqq A D^{p} f_{p}(\alpha+\varepsilon) \text {. }
$$

The left side does not depend on $\varepsilon$ and so inequality (1) holds for $K$.

2. To prove (1) for convex polyhedra $K$ we form, from a given $K$, four convex bodies $K_{1}, K_{2}, K_{3}, K_{4}$ for which

$$
S_{p}\left(K_{j}, \omega_{\alpha}\right) \leqq S_{p}\left(K_{j+1}, \omega_{\alpha}\right), j=1,2,3,
$$

and

$$
\begin{aligned}
& S_{p}\left(K_{1}, \omega_{\alpha}\right)=S_{p}\left(K, \omega_{\alpha}\right), \\
& S_{p}\left(K_{4}, \omega_{\alpha}\right)=A D^{p} f_{p}(\alpha) .
\end{aligned}
$$

As a matter of notation $\Pi_{j}(u)$ signifies the support plane to $K_{j}$ with outer unit normal $u$. We write $\partial P$ for the boundary of any set $P$. 
The body $K_{1}$ is to be the convex closure of $B\left(\omega_{\alpha}\right)$. Since

$$
\bigcup_{u \in \omega_{\alpha}}\left(K_{1} \cap \Pi_{1}(u)\right)=B\left(\omega_{\alpha}\right)
$$

(8) holds. Also $K_{1}$ is polyhedral.

Let $\mathfrak{S}_{1}(u)$ signify the half-space with outer normal $u$ which is bounded by $\Pi_{1}(u)$. Of course, for $u$ in $\omega_{\alpha}, \mathscr{F}_{1}(u)$ is the half-space with outer normal $u$ bounded by $\Pi(u)$. Since $\alpha<\pi / 2$, the intersection of those $\mathfrak{S}_{1}(u)$ for which

$$
(u, v) \leqq \cos \alpha
$$

is a convex polyhedron $K_{2} \supseteqq K_{1}$. Here $v$, as before, is the centre of $\omega_{\alpha}$; we write $\omega_{\alpha}^{\prime}$ for those $u$ on $\Omega$ which satisfy the last inequality. Clearly

and so

$$
\bigcup_{u \in \omega_{\alpha}^{\prime}}\left(K_{1} \cap \Pi_{1}(u)\right)=\bigcup_{u \in \omega_{\alpha}^{\prime}}\left(K_{2} \cap \Pi_{2}(u)\right)
$$

$$
S_{p}\left(K_{1}, \omega_{\alpha}^{\prime}\right)=S_{p}\left(K_{2}, \omega_{\alpha}^{\prime}\right) \text {. }
$$

On the other hand $K_{1} \subseteq K_{2}$ implies that

$$
S_{p}\left(K_{1}, \Omega\right) \leqq S_{p}\left(K_{2}, \Omega\right) \text {. }
$$

This is a consequence of the representation of these total area functions as mixed volumes and the known monotonicity of mixed volumes $V(K, \cdots, K, E, \cdots, E)$ in $K$, cf. [2]. The additinity of area functions, our last inequality and (10) yield (7) for $j=1$.

The rest of the proof is treated in separate sections. In $\S 3$ we describe a plane $I_{0}$ normal to $v$, which cuts $K$ so that $B\left(\omega_{\alpha}\right)$, and hence $K_{2}$, lies in one of the half-spaces determined by $\Pi_{0}$. Call this half-space $\mathfrak{S}_{0}$. We take $K_{3}$ to be the intersection of $\mathscr{S}_{0}$ with

$$
\cap \mathfrak{S}(u)=\cap \mathfrak{S}_{1}(u)
$$

where these intersections are taken over those $u$ in the common boundary of $\omega_{\alpha}$ and $\omega_{\alpha}^{\prime}$, i.e., those $u$ for which

$$
(u, v)=\cos \alpha \text {. }
$$

The body $K_{3}$ contains $K_{2}$. To determine $\Pi_{0}$ it is necessary to consider circular cones of the form

$$
\left(v, x-x_{0}\right)+\left\|x-x_{0}\right\| \sin \alpha \leqq 0 .
$$

The norm is Euclidean. The vertex of such a cone is $x_{0}$; the axial ray within the cone has the direction $-v$; these cones are translates 
of one another. We choose $x_{0}$ so that the resulting cone contains $K$ and the distance from $K$ to the plane

$$
\left(v, x-x_{0}\right)=0
$$

is as small as possible. We call this tangent cone $C$.

In $\S 4(7)$ is proved for $j=2$.

$K_{4}$ is $C \cap \mathfrak{S}_{0}$. This intersection is clearly a convex body which contains $K_{3}$. In $\S 5$ we prove (7) for $j=3$. Finally (9) follows from a direct calculation sketched in $\S 6$.

3. Let us introduce a Cartesian coordinate system with origin at the vertex $x_{0}$ of $C$ and such that $v=(-1,0, \ldots, 0)$. The description of $C$ takes the form

$$
x_{1} \geqq \tan \alpha\left(x_{2}^{2}+\cdots+x_{n}^{2}\right)^{1 / 2}
$$

and the distance from $K$, which is in $C$, to the plane $x_{1}=0$ is minimal. This means that each half-space

$$
u_{2} x_{2}+\cdots+u_{n} x_{n} \geqq 0
$$

must contain a point of $B\left(\omega_{\alpha}\right) \cap \partial C$ for the following reason. If $\partial K \cap \partial C$ had no points in (12), a small translation of $K$ in the direction $u$ would cause $\partial K \cap \partial C$ to be empty; a subsequent small translation in the direction $v$ would reduce the distance from $K$ to $x_{1}=0$. Hence (12) contains a point $x$ of $\partial C \cap \partial K$. The tangent plane to $\partial C$ at $x$ is a support plane of $\partial K$ and the outer normal to this support plane makes an angle of measure $\alpha$ with $v$, i.e., falls in $\omega_{\alpha}$. Thus $x$ is also in $B\left(\omega_{\alpha}\right)$ as asserted.

We define conical bodies $C_{1}$ and $C_{2}$ to be the intersection of $C$ with the half-spaces

$$
x_{1} \leqq D \tan \alpha, x_{1} \leqq 2 D \tan \alpha
$$

respectively.

We first prove that

$$
B\left(\omega_{\alpha}\right) \cap \partial C \subseteq C_{1} .
$$

Suppose to the contrary that there is a $y$ in $B\left(\omega_{\alpha}\right) \cap \partial C$ for which $y_{1}>D \tan \alpha$. Since the radius of the intersection of $C$ with

$$
x_{1}=D \tan \alpha
$$

is $D$, a ball of radius $D$, centred at $y$, lies in a half-space of the form

$$
u_{2} x_{2}+\cdots+u_{n} x_{n}<0
$$

for some $u$. As noted in the previous paragraph, there is a point $x$ 
in the complement of (14) which is in $B\left(\omega_{\alpha}\right) \cap \partial C$. This would give two points $x$ and $y$ in $K$ separated by a distance greater than the diameter $D$ of $K$. The contradiction establishes (13).

Next we demonstrate

$$
B\left(\omega_{\alpha}\right) \subseteq C_{2} .
$$

Again the proof is by contradiction. Imagine $z$ to be a point in $B\left(\omega_{\alpha}\right)$ for which $z_{1}>2 D \tan \alpha$. $z$ cannot be on the $x_{1}$-axis for the following reason. Let $I I$ be a support plane to $K$ which contains $z$. There must be a half-space of the form (12) in which the points of $\Pi \cap \partial C$ lie in the half-space

$$
x_{1}>2 D \tan \alpha .
$$

This implies that the points of $\partial K \cap \partial C$ which lie in (12) are at a distance exceeding $2 D$ from $z$ which, again, contradicts the fact that $D$ is the diameter of $K$.

Let $z^{\prime}$ be the point nearest to $z$ on the $x_{1}$-axis. Set

$$
u=\left(z-z^{\prime}\right) /\left\|z-z^{\prime}\right\| ;
$$

$u$ is orthogonal to $v$ and $z^{\prime}$ and so

$$
0<\left(u, z^{\prime}-z\right)=-(u, z) .
$$

Thus $z$ satisfies

$$
u_{2} z_{2}+\cdots+u_{n} z_{n}<0 .
$$

There is also a point $x$ of

$$
B\left(\omega_{\alpha}\right) \cap \partial C_{1}=B\left(\omega_{\alpha}\right) \cap \partial C_{2}
$$

in the complementary half-space. Therefore the distance $\|z-x\|$ must exceed the distance between $(2 D \tan \alpha, 0, \cdots, 0)$ and the intersection of $\partial C_{1}$ with the plane

$$
x_{1}=D \tan \alpha .
$$

That is to say

$$
\|z-x\|>\left(D^{2}+D^{2} \tan ^{2} \alpha\right)^{1 / 2}>D .
$$

This is impossible for $x$ and $z$ in $K$ which completes the proof of (15). The plane

$$
x_{1}=2 D \tan \alpha
$$

is the cutting plane $\Pi_{0}$ of $\S 2$; the conical convex body $C_{2}$ is $K_{4}$. 
4. From the definitions of $K_{2}$ and $K_{3}$ we see that their support planes $\Pi_{2}(u)$ and $\Pi_{3}(u)$ coincide whenever their outer normal directions $u$ are in $\omega_{\alpha}$. Hence for such $u$, since $K_{2} \subseteq K_{3}$,

$$
K_{2} \cap \Pi_{2}(u) \subseteq K_{3} \cap \Pi_{3}(u) ;
$$

there is certainly equality when $u$ is in the interior of $\omega_{\alpha}$. Inequality (7) for $j=2$ follows from the next lemma, to the proof of which this section is devoted.

Lemma. Let $K$ and $K^{\prime}$ be two convex polyhedral bodies whose support planes with outer normal direction $u$ are denoted by $\Pi(u)$ and $\Pi^{\prime}(u)$. If

$$
K \cap \Pi(u) \subseteq K^{\prime} \cap \Pi^{\prime}(u)
$$

for each $u$ in some Borel set $\omega$ of $\Omega$, then

$$
S_{p}(K, \omega) \leqq S_{p}\left(K^{\prime}, \omega\right), \text { for } p=1,2, \cdots, n-1 .
$$

We first require a description of $S_{p}(K, \omega)$ where $K$ is polyhedral. In this we follow work, as yet unpublished, of J. Zelver.

Consider a set of the form $K \cap \Pi(u)$; this is a $p$-face $e_{p}$ when $e_{p}$ lies in a $p$-dimensional flat but not in a $(p-1)$-dimensional flat. The outer unit normals to support planes of $K$ which contain $e_{p}$ sweep out a closed, geodesically convex set $\omega\left(e_{p}\right)$ on $\Omega$ which is in $\mathscr{B}$ and is $(n-p-1)$-dimensional. Throughout $\omega\left(e_{p}\right)$ we distribute mass with constant density $\lambda_{p}\left(e_{p}\right)$ equal to the $p$-dimensional volume of $e_{p}$. Thus if $\omega$ is any subset of $\omega\left(e_{p}\right)$ which is in $\mathscr{B}$ and if $\mu_{n-p-1}(\omega)$ is its $(n-p-1)$-dimensional volume, then the mass falling in $\omega$ is $\lambda_{p}\left(e_{p}\right) \mu_{n-p-1}(\omega)$. The representation we seek is

$$
S_{p}(K, \omega)=\sum_{*} \lambda_{p}\left(e_{p}\right) \mu_{n-p-1}\left(\omega \cap \omega\left(e_{p}\right)\right) /\left(\begin{array}{c}
n-1 \\
p
\end{array}\right),
$$

where the starred summation is taken over all $e_{p}$ in $\partial K$.

Consider the vector sum $K+t E$ and let $\Pi^{*}(u)$ signify its support plane with outer normal $u$. If $x^{\prime}$ is a point of

$$
(K+t E) \cap I I^{*}(u),
$$

then there is a unique point $x$ in $K \cap I I(u)$ such that

$$
x^{\prime}-x=t u \text {. }
$$

Suppose $e_{p}$ to be the face of lowest dimension which contains $x$ and let $\left\{\Pi\left(u^{\prime}\right)\right\}$ be the set of support planes of $K$ which contain $e_{p}$ where $u^{\prime}$ ranges over $\omega\left(e_{p}\right)$. We form 


$$
\bigcup_{*}\left\{(K+t E) \cap \Pi^{*}\left(u^{\prime}\right)\right\},
$$

where the starred union is taken over those $u^{\prime}$ in $\omega \cap \omega\left(e_{p}\right)$. If (20) is not empty, it is made up of points $x^{\prime}$ to each of which corresponds a unique $x$ on

$$
\bigcup_{*}\left(K \cap \Pi\left(u^{\prime}\right)\right)=e_{p}
$$

for which (19) holds. Thus (20) is the Cartesian product of $e_{p}$ with that part of the boundary of $t E$ which is swept out by rays whose directions are in $\omega \cap \omega\left(e_{p}\right)$. Therefore, empty or not, the $(n-1)$ dimensional measure of $(20)$ is

$$
t^{n-p-1} \lambda_{p}\left(e_{p}\right) \mu_{n-p-1}\left(\omega \cap \omega\left(e_{p}\right)\right) .
$$

We add up all such contributions to $S_{n-1}(K+t E, \omega)$ ard obtain the sum

$$
\sum_{p=1}^{n} t^{n-p-1} \sum_{*} \lambda_{p}\left(e_{p}\right) \mu_{n-p-1}\left(\omega \cap \omega\left(e_{p}\right)\right) .
$$

On the other hand, from the generalized Steiner formula [3, p. 31], we have

$$
S_{n-1}(K+t E, \omega)=\sum_{p=1}^{n} t^{n-p-1}\left(\begin{array}{c}
n-1 \\
p
\end{array}\right) S_{p}(K, \omega) .
$$

The comparison of coefficients of like powers of $t$ in these two representations of $S_{n-1}(K+t E, \omega)$ yields (18).

Choose $u$ in $\omega$; neither set in (16) is empty and so $\Pi(u)$ and $\Pi^{\prime}(u)$ share a common point, have the same normal direction and so coincide. We have

$$
K^{\prime} \cap \Pi(u)=e_{p}^{\prime}
$$

for some $p$. By (16) either $K \cap \Pi(u)$ is a face $e_{p}$ of the same dimension $p$ or this intersection is a face of lower dimension. In the latter case there is no contribution to the sum in (18), i.e., the left side of (17), whereas there would be a positive contribution to the right side of (17). In the former case, from (16) it follows that

$$
\lambda_{p}\left(e_{p}^{\prime}\right) \geqq \lambda_{p}\left(e_{p}\right) \text {. }
$$

Also

$$
\mu_{n-p-1}\left(\omega \cap \omega\left(e_{p}^{\prime}\right)\right)=\mu_{n-p-1}\left(\omega \cap \omega\left(e_{p}\right)\right) .
$$

To see this, we prove that the two argument sets in (22) coincide by showing that, for any $u$ in $\Omega$, we have $K \cap \Pi(u) \supseteq e_{p}$ if and only if $K^{\prime} \cap \Pi(u) \supseteqq e_{p}^{\prime}$. 
If $K^{\prime} \cap \Pi(u) \supseteqq e_{p}^{\prime}$, then $e_{p} \subseteq e_{p}^{\prime} \subseteq \Pi(u)$ and $e_{p}$ also lies in $\partial K$. Hence $e_{p}$ lies in $K \cap \Pi(u)$. Suppose $e_{p} \subseteq K \cap \Pi(u)$; then $e_{p}$ lies in $\Pi(u)$. Since $e_{p} \subseteq e_{p}^{\prime}$ by (16) and these two sets have the same dimensionality, any point $x$ in $e_{p}^{\prime}$ is a linear combination of $p+1$ suitable points in $e_{p}$. But, being such a combination of points in $\Pi(u), x$ must be in $\Pi(u)$. Thus $e_{p}^{\prime}$ is in both $\Pi(u)$ and $K^{\prime}$ and so in their intersection.

Substitution from (21) and (22) into the representation (18) as it applies to $K$ and $K^{\prime}$ proves (17).

5. Our next step is to prove (7) for $j=3$. We first settle the simplest case: $p=n-1$. It is clear from the construction of $K_{3}$ and $K_{4}$ that, for $i=3,4$ :

$$
\begin{gathered}
S_{n-1}\left(K_{i}, \Omega-\omega_{\alpha}\right)=S_{n-1}\left(K_{i},\{-v\}\right), \\
S_{n-1}\left(K_{i}, \omega_{\alpha}\right)=S_{n-1}\left(K_{i}, \partial \omega_{\alpha}\right),
\end{gathered}
$$

and

$$
S_{n-1}\left(K_{i}, \partial \omega_{\alpha}\right) \cos \alpha=S_{n-1}\left(K_{i},\{-v\}\right)
$$

Consequently

$$
S_{n-1}\left(K_{i}, \Omega\right)=(1+\cos \alpha) S_{n-1}\left(K_{i}, \omega_{\alpha}\right) \text {. }
$$

Since $K_{3} \subseteq K_{4}$ and $S_{n-1}(K, \Omega)$ is increasing in $K$, it follows that (7) holds for $j=3, p=n-1$. For the cases $1 \leqq p<n-1$ a more elaborate argument is needed.

We shall examine the behaviour of $S_{p}\left(K_{i}, \omega_{\alpha}\right)$ in $K_{i}$ by studying that of

$$
Q_{i}=\int_{\Omega-\omega_{\alpha}}(v, u) S_{p}\left(K_{i}, d \omega(u)\right), i=3,4 .
$$

These integrals will be reduced to iterated integrals. For this purpose we let $\Omega_{n-1}$ denote the set of $u$ on $\Omega$ which are orthogonal to $v$ and we form, for each $u$ in $\Omega_{n-1}$, the vectors

$$
u_{\lambda}=[(1-\lambda) u+\lambda(-v)] /\|(1-\lambda) u+\lambda(-v)\| \text {. }
$$

As before, $v$ is the centre of $\omega_{\alpha}$. We have

$$
\left(u_{\lambda}, v\right)=-\lambda /(\phi(\lambda))^{1 / 2},
$$

where

$$
\phi(\lambda)=1-2 \lambda+2 \lambda^{2}
$$

Also, if $s$ signifies arc length along the circle through $v$ and $u$, 


$$
d s / d \lambda=1 / \phi(\lambda)
$$

Define $\lambda_{\jmath}<0$ by

$$
-\lambda_{0}=\cos \alpha\left(\phi\left(\lambda_{0}\right)\right)^{1 / 2} \text {. }
$$

As $u$ passes over $\Omega_{n-1}$ and $\lambda$ over the interval $\lambda_{0}<\lambda<1, u_{\lambda}$ sweeps out

$$
\Omega-\omega_{\alpha}-\{-v\}
$$

For such $u$ and $\lambda$ :

$$
\Pi_{i}\left(u_{\lambda}\right) \cap K_{i}=\Pi_{i}(u) \cap \Pi_{0} \cap K_{i}=\Pi_{i}(u) \cap k_{i},
$$

where we have set

$$
k_{i}=K_{i} \cap \Pi_{0},
$$

and we recall that $\Pi_{0}$ is the support plane of $K_{i}$ with outer normal $-v$. If we view each $k_{i}$ as a nondegenerate convex body in the $(n-1)$-dimensional space $\Pi_{0}$, then the outer normals $u$ to $k_{i}$ fall in $\Omega_{n-1}$ and $k_{i}$ has area functions

$$
s_{1}\left(k_{i}, \eta\right), \cdots, s_{n-2}\left(k_{i}, \eta\right)
$$

defined over the Borel sets $\eta$ of $\Omega_{n-1}$.

We write $Q_{i}$ as an iterated integral

$$
\int_{\lambda_{0}}^{1} \frac{-\lambda}{(\phi(\lambda))^{1 / 2}}\left(\int_{\Omega_{n-1}} s_{p}\left(k_{i}, d \eta(u)\right)\right) \frac{d \lambda}{\phi(\lambda)}=g S_{p}\left(k_{i}, \Omega_{n-1}\right)
$$

where

$$
g=\int_{\lambda_{0}}^{1} \frac{-\lambda d \lambda}{(\phi(\lambda))^{3 / 2}}<0
$$

Here we have used the fact that the point $-v$ can be deleted from $\Omega-\omega_{\alpha}$ without affecting $Q_{i}$ in virtue of (3) and the assumption that $p<n-1$. Since $k_{3} \subseteq k_{4}$

$$
s_{p}\left(k_{3}, \Omega_{n-1}\right) \leqq s_{p}\left(k_{4}, \Omega_{n-1}\right)
$$

and, from the negativity of $g$, it follows that

$$
Q_{3} \geqq Q_{:} \cdot
$$

The first condition in (2), which is satisfied by any area function, shows that

$$
Q_{i}+\int_{\omega_{\alpha}}\left(v, u_{\lambda}\right) S_{p}\left(K_{i}, d \omega\left(u_{\lambda}\right)\right)=0
$$

Hence, from our last inequality, we obtain 
(23)

$$
\int_{\omega_{\alpha}}\left(v, u_{\lambda}\right) S_{p}\left(K_{3}, d \omega\left(u_{\lambda}\right)\right) \leqq \int_{\omega_{\alpha}}\left(v, u_{\lambda}\right) S_{p}\left(K_{4}, d \omega\left(u_{\lambda}\right)\right) .
$$

Let $x_{0}$ signify the vertex of the cone $K_{4}$ and denote by $\omega_{\alpha}^{0}$ the interior of $\omega_{\alpha}$. Then for all $u$ in $\omega_{\alpha}^{0}$

$$
K_{4} \cap \Pi_{4}(u)=x_{0}
$$

and, because $p \geqq 1$,

$$
S_{p}\left(K, \omega_{\alpha}^{0}\right)=0 .
$$

Therefore on the right side of (23) the integration needs to be extended only over $\partial \omega_{\alpha}$ throughout which $\left(v, u_{\lambda}\right)$ is $\cos \alpha$. This yields for the right side of (23)

$$
\cos \alpha S_{p}\left(K_{4}, \omega_{\alpha}\right) \text {. }
$$

Consider the left side of (23). For $u_{2}$ in $\omega_{\alpha}$ we have

$$
\left(v, u_{2}\right) \geqq \cos \alpha
$$

and so we may strengthen inequality (23) by replacing the left side by

$$
\cos \alpha S_{p}\left(K_{3}, \omega_{\alpha}\right) \text {. }
$$

After division by $\cos \alpha$ the strengthened inequality is just (7) for $j=3,1 \leqq p<n-1$.

6. It remains to prove (9). In the Cartesian coordinate system of section three, $K_{4}$ is the set of points $x$ for which

$$
\tan \alpha\left(x_{2}^{2}+\cdots+x_{n}^{2}\right)^{1 / 2} \leqq x_{1} \leqq 2 D \tan \alpha .
$$

Let $t E^{*}$ be the convex body formed by the intersection of the"ball $t E$ with the reflected polar cone to $C$, i.e.,

$$
x_{1} \leqq-\operatorname{ctn} \alpha\left(x_{2}^{2}+\cdots+x_{n}^{2}\right)^{1 / 2} .
$$

The vector sum $K_{4}+t E^{*}$ is a convex body of revolution whose radial distance $r(\xi)$ in the plane $x_{1}=\xi$ has the representation

$$
\begin{aligned}
r(\xi) & =\left(t^{2}-\xi^{2}\right)^{1 / 2},-t \leqq \xi \leqq-t \cos \alpha ; \\
& =\xi \operatorname{ctn} \alpha+t c s c \alpha,-t \cos \alpha \leqq \xi \leqq 2 D \tan \alpha-t \cos ^{-} \alpha ; \\
& =2 D \sec ^{2} \alpha-\xi \tan \alpha, 2 D \tan \alpha-t \cos \alpha \leqq \xi \leqq 2 D \tan \alpha .
\end{aligned}
$$

The volume $V\left(K_{4}+t E^{*}\right)$ is

$$
\omega_{n-1} \int_{-t}^{2 D \tan \alpha} r^{n-1}(\xi) d \xi /(n-1) .
$$


Here $\omega_{n-1}$ is the area of the unit spherical surface in Euclidean $(n-1)$-dimensional space and is given by

$$
\omega_{n-1}=2 \pi^{(n-1) / 2} / \Gamma((n-1) / 2),
$$

where $\Gamma$ is the usual gamma function.

We equate (25) with the Steiner polynomial

$$
V\left(K_{4}+t E^{*}\right)=\sum_{p=0}^{n}\left(\begin{array}{l}
n \\
p
\end{array}\right) t^{n-p} V_{p}\left(K_{4}, E^{*}\right),
$$

where $V_{p}\left(K_{4}, E^{*}\right)$ is the mixed volume

$$
V(\underbrace{K_{4}, \cdots, K_{4}}_{p}, \underbrace{E^{*}, \cdots, E^{*}}_{n-p}) .
$$

Substitution from (24) into (25) and a comparison of coefficients of like powers of $t$ yields

$$
V_{p}\left(K_{4}, E^{*}\right)=\omega_{n-1}(2 D)^{p}(\sin \alpha)^{n-p-1} \sec \alpha / n(n-1) .
$$

We consider next the brush set (Bürstenmenge) $B_{t}\left(K_{4}, \omega_{\alpha}\right)$ which is formed from $K_{4}$ in the following manner. At each point $x$ of

$$
\bigcup_{u \in \omega_{\alpha}}\left(K_{4} \cap \Pi_{4}(u)\right)
$$

we draw all segments $x+\theta u, 0<\theta \leqq t$, corresponding to $u$ in $\omega_{\alpha}$. The union of these segments is $B_{t}\left(K_{4}, \omega_{\alpha}\right)$. Clearly this is

$$
\left(K_{4}+t E^{*}\right)-K_{4}
$$

and so the volume $V_{t}\left(K_{4}, \omega_{\alpha}\right)$ of $B_{t}\left(K_{4}, \omega_{\alpha}\right)$ is

$$
V\left(K_{4}+t E^{*}\right)-V\left(K_{4}\right)=\sum_{p=0}^{n-1}\left(\begin{array}{l}
n \\
p
\end{array}\right) t^{n-p} V_{p}\left(K_{4}, E^{*}\right) .
$$

On the other hand, ef. [3, p. 31],

$$
V_{t}\left(K_{4}, \omega_{\alpha}\right)=\sum_{p=0}^{n-1}\left(\begin{array}{c}
n \\
p
\end{array}\right) t^{n-p} S_{p}\left(K_{4}, \omega_{\alpha}\right) / n
$$

A comparison of coefficients of like powers of $t$ in these two representations of $V_{t}\left(K_{4}, \omega_{\alpha}\right)$ yields

$$
S_{p}\left(K_{4}, \omega_{\alpha}\right)=n V_{p}\left(K_{4}, E^{*}\right)
$$

and this, together with (26), gives (9) with

$$
A=2^{p} \omega_{n-1} /(n-1) \text {. }
$$

This completes the proof of (1). 


\section{REFERENCES}

1. A. D. Aleksandrov, Zur Theorie der gemischten Volumina von konvexen hörper III, Die Erweiterung zweier Lehrsätze Minkowskis uber die konvexen Polyeder auf beliebige konvexe Flächen, Mat. Sbornik, N.S. 3 (1938), 27-46; (Russian with German summary).

2. H. Busemann, Convex Surfaces, New York, 1958.

3. W. Fenchel and B. Jessen, Mengenfunktionen und konvexe Körper, Det Kgl. Danske Videnskab, Selskab, Math-fys. Medd. 16 (1938), 3.

4. W. J. Firey, The determination of convex bodies from their mean radius of curvature functions, Mathematika, 14 (1967), 1-13.

5. W. J. Firey, Christoffel's problem for general convex bodies, Mathematika 15 (1968), $7-21$.

Received June 6, 1968, and in revised form December 30, 1969.

OREgon State UNIVERSity

Corvallis, Oregon 



\section{PACIFIC JOURNAL OF MATHEMATICS}

\section{EDITORS}

\author{
H. SAMELSON \\ Stanford University \\ Stanford, California 94305

\section{Richard Pierce} \\ University of Washington \\ Seattle, Washington 98105
}

J. DugundJI

Department of Mathematics

University of Southern California

Los Angeles, California 90007

RichaRd ARENS

University of California

Los Angeles, California 90024

\section{ASSOCIATE EDITORS}
E. F. BECKENBACH
B. H. NeUMANN
F. WOLE
K. YoshidA

\section{SUPPORTING INSTITUTIONS}

\author{
UNIVERSITY OF BRITISH COLUMBIA \\ CALIFORNIA INSTITUTE OF TECHNOLOGY \\ UNIVERSITY OF CALIFORNIA \\ MONTANA STATE UNIVERSITY \\ UNIVERSITY OF NEVADA \\ NEW MEXICO STATE UNIVERSITY \\ OREGON STATE UNIVERSITY \\ UNIVERSITY OF OREGON \\ OSAKA UNIVERSITY \\ UNIVERSITY OF SOUTHERN CALIFORNIA
}

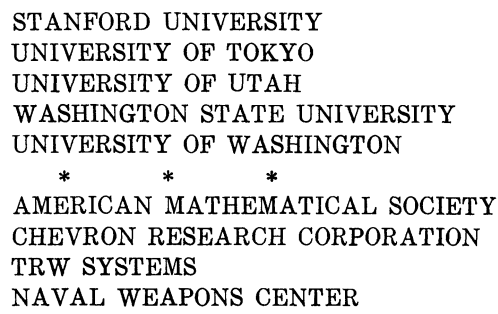

The Supporting Institutions listed above contribute to the cost of publication of this Journal, but they are not owners or publishers and have no responsibility for its content or policies.

Mathematical papers intended for publication in the Pacific Journal of Mathematics should be in typed form or offset-reproduced, (not dittoed), double spaced with large margins. Underline Greek letters in red, German in green, and script in blue. The first paragraph or two must be capable of being used separately as a synopsis of the entire paper. The editorial "we" must not be used in the synopsis, and items of the bibliography should not be cited there unless absolutely necessary, in which case they must be identified by author and Journal, rather than by item number. Manuscripts, in duplicate if possible, may be sent to any one of the four editors. Please classify according to the scheme of Math. Rev. Index to Vol. 39. All other communications to the editors should be addressed to the managing editor, Richard Arens, University of California, Los Angeles, California, 90024.

50 reprints are provided free for each article; additional copies may be obtained at cost in multiples of 50 .

The Pacific Journal of Mathematics is published monthly. Effective with Volume 16 the price per volume (3 numbers) is $\$ 8.00$; single issues, $\$ 3.00$. Special price for current issues to individual faculty members of supporting institutions and to individual members of the American Mathematical Society: $\$ 4.00$ per volume; single issues $\$ 1.50$. Back numbers are available.

Subscriptions, orders for back numbers, and changes of address should be sent to Pacific Journal of Mathematics, 103 Highland Boulevard, Berkeley, California, 94708.

PUBLISHED BY PACIFIC JOURNAL OF MATHEMATICS, A NON-PROFIT CORPORATION

Printed at Kokusai Bunken Insatsusha (International Academic Printing Co., Ltd.), 7-17, Fuj̣imi 2-chome, Chiyoda-ku, Tokyo, Japan. 


\section{Pacific Journal of Mathematics}

\section{Vol. 35, No. $2 \quad$ October, 1970}

Valentin Danilovich Belousov and Palaniappan L. Kannappan, Generalized Bol functional equation .................................... 259

Charles Morgan Biles, Gelfand and Wallman-type compactifications ........... 267

Louis Harvey Blake, A generalization of martingales and two consequent convergence theorems .................................... 279

Dennis K. Burke, On p-spaces and $w \Delta$-spaces..................... 285

John Ben Butler, Jr., Almost smooth perturbations of self-adjoint operators . . . . . . 297

Michael James Cambern, Isomorphisms of $C_{0}(Y)$ onto $C(X) \ldots \ldots \ldots \ldots \ldots . \ldots 307$

David Edwin Cook, A conditionally compact point set with noncompact closure ... 313

Timothy Edwin Cramer, Countable Boolean algebras as subalgebras and homomorphs .........................................

John R. Edwards and Stanley G. Wayment, A v-integral representation for linear operators on spaces of continuous functions with values in topological vector spaces.............................................

Mary Rodriguez Embry, Similarities involving normal operators on Hilbert

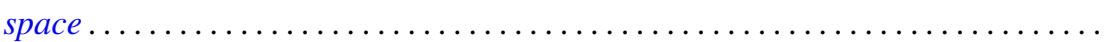

Lynn Harry Erbe, Oscillation theorems for second order linear differential

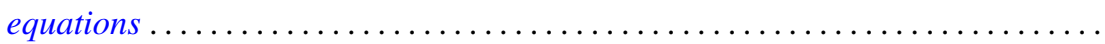

William James Firey, Local behaviour of area functions of convex bodies .......... Joe Wayne Fisher, The primary decomposition theory for modules ..............

Gerald Seymour Garfinkel, Generic splitting algebras for Pic ..................

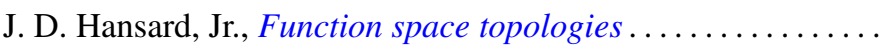

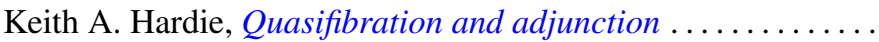

G. Hochschild, Coverings of pro-affine algebraic groups ...........

Gerald L. Itzkowitz, On nets of contractive maps in uniform spaces ..

381

389

399

417

Melven Robert Krom and Myren Laurance Krom, Groups with free nonabelian subgroups....................................

James Robert Kuttler, Upper and lower bounds for eigenvalues by finite differences ......................................

Dany Leviatan, A new approach to representation theory for convolution transforms . . .

Richard Beech Mansfield, Perfect subsets of definable sets of real numbers ...

Brenda MacGibbon, A necessary and sufficient condition for the embedding of a

Lindelof space in a Hausdorff $\mathscr{H} \sigma$ space ..................

David G. Mead and B. D. McLemore, Ritt's question on the Wronskian ....

Edward Yoshio Mikami, Focal points in a control problem .....

Paul G. Miller, Characterizing the distributions of three independent n-dimensional random variables, $X_{1}, X_{2}, X_{3}$, having analytic characteristic functions by the joint distribution of $\left(X_{1}+X_{3}, X_{2}+X_{3}\right)$. . .

P. Rosenthal, On the Bergman integral operator for an elliptic partial differential equation with a singular coefficient....

Douglas B. Smith, On the number of finitely generated $O$-group 